\title{
Comparative method for actuarial analysis of cardiac valve replacements
}

\author{
ENDRE BODNAR, STEVEN HABERMAN, AND WILLIAM H. WAIN \\ From the Department of Surgery, Cardiothoracic Institute, London; Department of Mathematics, \\ The City University, London; and Homograft Department, National Heart Hospital, London
}

SUMMARY The use of survival analysis in cardiac surgical reports has been widely accepted in order to characterise and compare performances of different valve prostheses. However, the analyses that have been described elsewhere may be improved in several respects. The original method proposed for prosthetic valve evaluation examined follow-up related to patients rather than the valves themselves and therefore neglected the possibility of factors other than the valve itself which could affect the fate of cardiac patients. Furthermore, there has not yet been any application of life table methods to compare two different sets of data, or to estimate the average time before an event occurs.

The present work defines valve function and malfunction and separates patient survival from valve performance. We define statistical indices, namely the median remaining lifetime and the instantaneous decrement rate. Both indices can be useful; the former measures the average survival time and the latter focuses attention on the intensity of risk changes. Using established statistical theory we calculate the significance level of difference between two independent survival rates. We qualify the presentation of probabilities with a statement of the associated standard errors.

Statistical analysis based on the life table has been proposed as an objective method for the evaluation of long-term results in cardiac surgery (Anderson et al., 1974; Grunkemeier et al., 1975). This method offers an accurate and simple means for estimating the prognosis of patients with chronic disease. The method has been used for many years to determine survival rates of cancer victims. Several authors have provided comprehensive descriptions (Berkson and Gage, 1950; Merrell and Shulman, 1955; Cutler and Ederer, 1958; Axtell, 1963). The approach was called the 'actuarial method' by Berkson and Gage (1950) who used a formula first proposed by Frost (1933), recognising that it had been widely used by actuaries since the 19th century as an application of the theory of 'multiple decrements' (Benjamin and Haycocks, 1970). Consideration of these problems first started with Daniel Bernoulli's discussion of the smallpox inoculation problem which is reviewed by Karn (1931).

\section{Calculation of patient survival}

We shall consider the application of actuarial analysis to follow-up studies where the population under investigation has undergone a valve opera-

Received for publication 1 March 1979 tion. We shall define patient survival and valve performance to be separate entities. Firstly, we shall discuss the method for assessing patient survival, and then extend the results, in a later section, to assessing valve performance.

In measuring the proportion of patients alive $\mathbf{n}$ years (say, $n=5$ ) after the operation, an important advantage of the actuarial method is that it uses all the information collected up to the time of analysis (that is the closing date of the study). Thus, one need not restrict one's attention to only those persons whose operation occurred more than five years before the closing date. Those entering four, three, two, and one year earlier contribute much relevant information to the evaluation of the probability of surviving five years. This extra information is utilised by pooling the data from groups of patients entering the study at different times.

The increased precision in estimating survival probabilities has been documented (Merrell and Shulman, 1955; Cutler and Ederer, 1958). This advantage is particularly relevant to surgical studies where the bulk of experience is concentrated at the earliest durations though the surgeon may be particularly interested in results at the longest duration after the operation.

At the closing date of such a follow-up study, patients are likely to have been observed for varying 
lengths of time, some having had the operation a long time previously, others more recently. Some patients will have died, the time of death being usually known accurately (with other end points, for example the occurrence of valve complications, the precise time of the event may not be known). Other patients are known to be alive, and would have survived further had the study been extended. Some may have been lost to follow-up for various reasons between successive examinations. Others may have been removed from the study for medical reasons (perhaps by the intervention of another disease or death from a different cause).

These last three groups, viz. (a) those who would have continued had not the closing date of the study intervened; (b) those who have been lost to followup; and (c) those who have been removed from the study, create a common category called 'withdrawals'. The combined group is of prime importance since it is usually part of the data, and it is this which makes the actuarial method of probability calculation necessary. If there were no withdrawals and each patient was observed until their time of death, the calculation of survival probabilities at different times after operation would be simple. Thus, the $\mathbf{n}$ year probability of surviving would be the same as the proportion of patients with survival times greater than $\mathrm{n}$. The problems would be reduced to ascertaining the distribution of survival times.

The mathematics of the actuarial method are not very difficult but it is not necessary for clinicians to understand the theory which is well established. This is set out in Appendix I.

Previous cardiac surgical reports which have discussed the use of the actuarial method have not considered the problem of comparing two sets of survival data. We present in Appendix I a method for testing how statistically significant is the difference between two independent sets of data.

In addition we introduce the instantaneous probability of dying which characterises the change in the intensity of risk, and is very useful for drawing attention to points where there is a dramatic change in experience. We also introduce the median remaining life-time which measures the average survival time.

These techniques are described in detail in Appendix I in terms of patient survival. However, they may also be used for measuring valve performance, and will be mentioned in the following sections.

\section{Calculation of valve performance}

When introducing the actuarial method to cardiac surgery, Anderson et al. (1974) proposed the assessment of patient survival after the insertion of a given prosthetic valve. They assumed that postoperative survival and/or complication-free survival was predetermined by the quality of the given valve prosthesis. Though such an assumption is reasonable when the main risk factor during the whole followup period is the valve, it neglects the possibility of an excellent prosthesis when the fate of patients will be determined by factors other than the prosthesis itself. Moreover, the method of constructing the life tables for cardiac surgical patients has been designed to assess follow-up not on a condition, such as a valve, but on the carrier of that condition, the patient. Thus, the straightforward interpretation of data on patient follow-up as a characteristic of valve performance can be misleading. Nevertheless, patient survival and valve performance can be separated, and given independent consideration.

The risk attributed to biological, prosthetic, or bioprosthetic cardiac valves is caused by one or more of five main malfunction categories as follows:

(1) Mechanical malfunction. Stenosis and/or regurgitation, as defined in the NYHA definition of cardiac anomalies, and irrespective of the pathomorphology of stenosis and regurgitation involving paravalvar leaks, or a haemodynamically significant gradient across an otherwise wellfunctioning prosthetic or bioprosthetic valve.

(2) Infection. Involvement of the valve in septic conditions, described as acute or subacute endocarditis by the NYHA definition.

(3) Thrombotic apposition on valve surface with or without consecutive embolism. Embolism in the relevant side of the circulation with or without thrombotic apposition on the valve.

(4) Pathological bleeding caused by anticoagulation resulting from prosthetic valve.

(5) Haemolysis. Clinically manifest haemolysis in the presence of a given valve and without coexisting haematological disease.

In terms of actuarial analysis any valve can be free of fatal malfunction, that is the patient is either alive or dead and the death is neither caused by nor accompanied by any of the above mentioned five conditions. Next, the valve can be functioning until the cessation of function, which means either until a valve-related death or the removal of the valve and its replacement by another cardiac prosthesis. Finally the valve can function without complications corresponding to the criterion that none of the above mentioned five conditions has yet occurred (Fig. 1).

Thus, the meaning of complication is both cumulative and comprehensive involving all of the possible malfunctions attributed to the given valve.

Actuarial analysis can be adapted to the analysis 


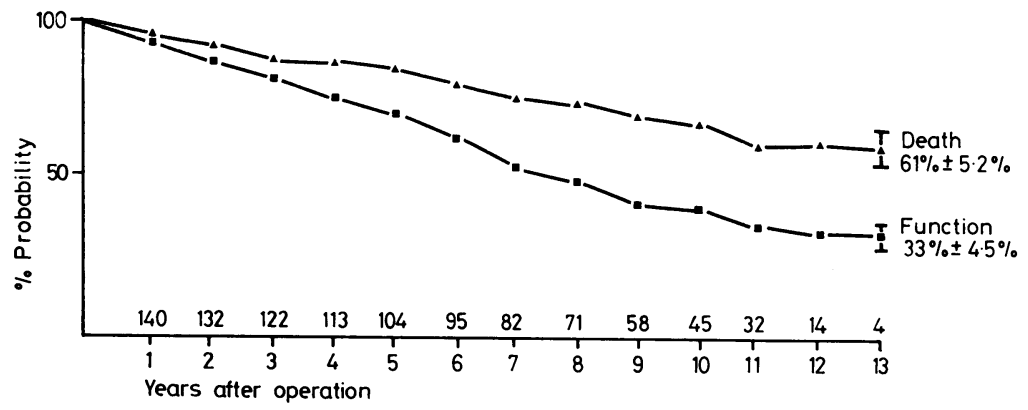

Fig. 1 Freeze dried homograft valves. Probability of valve related death and valve function without complication (cumulative). of non-fatal events (for example, complications), by considering such events as terminal, that is by treating the experience which individuals or valves contribute to the study, in the same way as death is treated in Appendix I.

When assessing valve performance, the withdrawal category will comprise the following three groups of valves, viz.

(a) Those which would have continued had not the closing date of the study intervened.

(b) Those which have been lost to follow-up.

(c) Those who die and the cause is either not related to the valve or is unknown.

The life tables for assessing valve performance are constructed in an analogous way to that described in Appendix I for patients and in Appendix II we present a worked example. For convenience, in the definitions below, we use terms like 'death' or 'loss' to refer to valves, even though it is the patient who dies or who is lost.

Consider the interval between $\mathrm{x}$ and $\mathrm{x}+1$ years after the operation.

Let $L_{x}$ be the number of originally inserted valves functioning at the beginning of the interval.

Let $D_{x}$ be the number of deaths, caused by or accompanied by any of the above five malfunctions, occurring in the interval.

Let $\mathbb{W}_{\mathbf{x}}$ be the number of valves withdrawn during the interval.

Let $R_{x}$ be the number of valves removed at successful re-operation during the interval.

Let $C_{x}$ be the number of valve complications involving neither death nor reoperation during the interval.

Then $L_{x+1}=L_{x}-\left(D_{x}+W_{x}+R_{x}+C_{x}\right)$ and a life table may be constructed.

Three different survival curves will be derived relating to free of fatal malfunction, free of malfunction, and free of complication. These will be considered in turn.

\section{FREE OF FATAL MALFUNCTION}

Let $E_{\mathbf{z}}{ }^{d}$ be the number of valves exposed to the risk of death caused or accompanied by any of the five malfunctions during the interval.

Then $E_{x}{ }^{d}$ is not equivalent to $L_{x}$ since the withdrawals, removals, and complications who have not completed the interval of observation under consideration must not be credited with having done so. In order to reach an expression for $\mathrm{E}_{\mathrm{x}}{ }^{\mathrm{d}}$, we shall assume that these valves withdrawing $\left(W_{x}\right)$, reoperated $\left(\mathbf{R}_{\mathbf{x}}\right)$, or experiencing complications $\left(\mathbf{C}_{\mathbf{x}}\right)$ in the interval $x$ to $x+1$ are exposed to the risk of dying from a valve related cause for half the interval, so that

$$
\mathrm{E}_{\mathbf{x}}{ }^{\mathrm{d}}=\mathrm{L}_{\mathrm{x}}-\frac{1}{2}\left(\mathrm{~W}_{\mathbf{x}}+\mathrm{R}_{\mathbf{x}}+\mathrm{C}_{\mathrm{x}}\right) \text {. }
$$

The actuarial estimate of the probability of a valve related death in the interval $x$ to $x+1$ for a valve alive and functioning at $x$ is $q_{x}{ }^{d}$ where

$$
q_{x}{ }^{d}=\frac{D_{x}}{E_{x}}
$$

Given $\mathrm{q}_{\mathrm{x}}{ }^{\mathrm{d}}$, formulae may be set up, as in Appendix I, for $P_{x}{ }^{d}$, cumulative probability of surviving free of a valve related death from $o$ to $x$; $\mu_{x+1} d$, the instantaneous valve related death rate at $\mathrm{x}+\frac{1}{2} ; \mathbf{m}_{\mathbf{x}}{ }^{\mathrm{d}}$, the median remaining lifetime for a valve free of fatal malfunction at time $x$ after operation; and the corresponding standard errors.

These functions are estimates of the values that would have been obtained if the valve removals, complications, and withdrawals were eliminated as types of departure from the study.

\section{FREE OF MALFUNCTION}

Let $E_{x} d r$ be the number of valves exposed to the risk of malfunction during the interval $x$ to $x+1$.

Then, after a similar argument to the above with corresponding assumptions, one can show that $\mathbf{E}_{\mathbf{x}}{ }^{\mathrm{d} \mathbf{r}}=\mathbf{L}_{\mathbf{x}}-\frac{1}{2}\left(\mathbf{W}_{\mathbf{x}}+\mathbf{C}_{\mathbf{x}}\right)=\mathbf{E}_{\mathbf{x}}{ }^{\mathrm{d}}+\frac{1}{2} \mathbf{R}_{\mathbf{x}}$.

Then the actuarial estimate of the probability of a malfunction occurring in the interval $x$ to $x+1$ for a valve alive and functioning at $x$ is $q_{x}{ }^{d r}$ where

$$
q_{x}{ }^{d r}=\frac{D_{x}+R_{x}}{E_{x} d r}
$$

Given $q_{x}{ }^{d r}$, formulae may be set up, as above, for $P_{x}{ }^{d r}$, the cumulative probability of surviving 
free of a malfunction from o to $x ; \mu_{x+1} d r$, the instantaneous malfunction rate at time $x+\frac{1}{2} ; m_{x}{ }^{d r}$, the median lifetime remaining for a valve free of a malfunction at time $\mathrm{x}$ after operation; and the corresponding standard errors.

\section{FREE OF COMPLICATION}

Let $E_{x}$ arc be the number of valves exposed to the risk of complication during the interval $x$ to $x+1$. Then, after a similar argument to the above with corresponding assumptions, one can show that $E_{x} d r c=L_{x}-\frac{1}{2} W_{x}=E_{x} d r+\frac{1}{2} C_{x}$.

Then the actuarial estimate of the probability of a complication occurring in the interval $x$ to $x+1$ for a valve alive and functioning at $x$ is $q_{x}{ }^{d r c}$ where

$q_{x}^{d r c}=\frac{D_{x}+R_{x}+C_{x}}{E^{d r c}}$

Given $\mathrm{q}_{\mathrm{x}}{ }^{\mathrm{drc}}$, formulae may be set up, as above, for $P_{x} d r c$, the cumulative probability of surviving free of a complication from o to $\mathrm{x} ; \mu^{\mathrm{drc}} \mathbf{x + y}$, the instantaneous complication rate at time $x+\frac{1}{2} ; m_{x} d r c$, the median remaining lifetime for a valve free of complication at time $x$ after operation, and the corresponding standard errors.

INDIVIDUAL AND CUMULATIVE

EVENT-FREE CURVES

The quality of life may be analysed by the actuarial method considering not only death but also possible complications, as described above. Two fundamentally different methods have been proposed by the Oregon University Group: the individual complication-free survival curve (Anderson et al., 1974), for example $\mathbf{P}^{\mathrm{d}}, \mathrm{Pr}^{\mathrm{r}}, \mathrm{P}^{\mathrm{c}}$, and the cumulative complication-free survival curve (Grunkemeier et al., 1975), for example $\mathbf{P d r}, \mathbf{P d r c}^{\mathrm{dr}}$.

The term 'cumulative' is thus used in two senses. Firstly it is used to refer to the combining of complications (or, in general, decrements) and secondly it is used to refer to the combining of successive years after the operation. This dual use of the same word is confusing. We suggest a more suitable nomenclature would be use of the term 'multiple complication' (and, in general, 'multiple decrement'), for the first purpose, and to use 'cumulative' for the second purpose only. So individual event-free curves could be described as 'single decrement'.

The individual event-free calculation (that is single decrement) analyses only one selected complication and assumes that no other complication can occur, not even death of the patient. Calculating each individual event-free complication curve (for example, embolism, endocarditis) will involve the death being included in the withdrawn category (as above) and thus contributing a half-year event-free survival.

Because of this disadvantage, Grunkemeier et al. proposed the cumulative event-free survival curve (multiple decrement) which considers death or complication as a final event, whichever occurs first. Not only one, but two, three, or all the possible complications can be analysed in this way. Whichever occurs first, death or any of the complications will count as a final, terminating event, and the rest of the patients will experience an event-free survival.

The reasonable difference between the two methods can be well illustrated on the follow-up of patients, or, as in this case, on valve performance (see Appendix II). This is based on 140 isolated aortic valve replacements between 1964 and 1967 at the National Heart Hospital. There was not a single embolism, 87 per cent of the valves were free of technical failure, 83 per cent free of infective disease, and 51 per cent free of degenerative failure after 13 years.

Not surprisingly, the cumulative event-free survival curve (multiple decrement) creates another impression. There are only 33 per cent of the valves functioning free of complication. In fact, the freeze drying of the homografts was abandoned well before actuarial analysis could have come into effect. Obviously, the conclusion can be drawn that fair, independent judgement on a valve and on its overall performance can scarcely be made without considering the cumulative complication-free survival curve (multiple decrement).

None the less, the individual event-free curves (single decrement) can be very useful for the exexpression of the composition of complications as well as their relation to each other. In this way it becomes easy to show that technical failures cause an early cessation in valve performance, that infection has not been recorded after the first $\mathbf{1 0}$ years, and that degeneration begins later but rises steadily.

Certainly, in this case the comment would be 33 per cent event-free valve function after 13 years, with some early technical failure, later a continuing degeneration as well as some infection causing malfunction.

The danger of considering individual event-free curves (single decrement) in assessment of patient survival or valve performance is increased when there is no single major risk factor, but the complications are caused by several lesser ones. The danger increases in proportion to the number of complication-groups taken into account. Ten individual complications each with a 90 per cent complication-free survival can easily mean that neither a single patient nor valve survives without 
complication. Thus, the cumulative event-free survival function (multiple decrement) should be the primary approach to an actuarial analysis in the assessment of valve performance.

\section{RELATION BETWEEN SURVIVAL CURVES}

If the above arguments had been used to derive estimates of the probability of the occurrence of a valve removal $\mathrm{q}_{\mathrm{x}}{ }^{\mathrm{r}}$ or the probability of the occurrence of a non-fatal complication involving no removal $\mathrm{q}_{\mathrm{x}} \mathrm{c}$, we could construct a free of removal survival curve $P_{x}{ }^{r}$ and a free of non-fatal complication survival curve $P_{x}{ }^{c}$. Then it may be shown that (Benjamin and Haycocks, 1970): (a) the instantaneous probability of a complication occurring is equal to the sum of the three single decrement instantaneous probabilities, that is

$$
\mu^{\mathrm{drc}}=\mu^{\mathrm{d}}+\mu^{\mathrm{r}}+\mu^{\mathrm{c}}
$$

and (b) the probability of surviving without a complication is equal to the product of the three single decrement probabilities of surviving, that is

$$
\mathbf{P d}^{\mathrm{rc}}=\mathbf{P}^{\mathrm{d}} . \mathbf{P r} \text {. Pc. }
$$

Thus the instantaneous probabilities combine together in an additive way, and the survival curves combine together in a multiplicative way.

Because of this multiplicative property, it may be shown that the difference between two survival curves has a simple interpretation (Grunkemeier et al., 1975). For example, the difference $P_{x} d-P_{x} d r$ (between the survival curve free of valve-related deaths and the survival curve free of malfunction) represents the probability of a patient being alive at time $\mathrm{x}$ but with a valve replaced. However, no information is provided about the subsequent experience of such patients with replaced valves.

In actuarial mathematics, functions like $\mathbf{P r}, \mathrm{Pd}^{\mathrm{d}}$, and $\mathrm{P}^{\mathrm{c}}$ are called single decrement functions, and those like Prd and Prcd are called multiple decrement functions. Thus, these two factors must be given separate assessments to achieve unbiased statistical analysis.

We acknowledge the invaluable help given by Professor Bernard Benjamin, City University, and by Mr Donald N. Ross, National Heart Hospital.

\section{Appendix I}

A particular feature of the actuarial method is that the time since operation is divided into intervals of convenient length. Without loss of generality, we shall consider the case where the intervals are of length one year.

If we let $\mathrm{x}$ denote the number of years since operation, and if we consider the year of followup between $x$ and $x+1$, then

let $l_{x}$ be the number of patients alive at the beginning of the interval let $d_{x}$ be the number of deaths during that interval let $w_{\mathbf{x}}$ be the number of withdrawals during that interval (i.e. number of patients lost, number of patients removed, and number of patients alive at the closing date which intervened during that interval).

Then $l_{x+1}=l_{x}-d_{x}-w_{x}$. And a life table may be constructed.

Let $E_{x}$ be the effective number of patients exposed to the risk of dying during that interval.

Then $E_{x}$ is not equivalent to $l_{x}$, since the withdrawals, $w_{\mathbf{x}}$, who have not completed the interval of observation under consideration must not be credited with having done so. All living patients will be found surviving an incomplete interval in their latest year of follow-up except those rare cases whose anniversary of operation coincides with the closing date of the study or date of loss or removal.

In order to reach an expression for $E_{\mathbf{x}}$ we need to make some assumptions about the times of withdrawals for the $w_{x}$ group. We shall assume that the withdrawn patients in the interval $x$ to $x+1$ are exposed to the risk of dying on average for half that interval, so that

$$
\mathrm{E}_{\mathbf{x}}=\mathbf{l}_{\mathbf{x}}-\frac{1}{2} \mathbf{w}_{\mathbf{x}} \text {. }
$$

Then, the actuarial estimate of the probability of dying in the interval $x$ to $x+1$ for a patient alive at $x$ is $q_{x}$ where

$$
\mathrm{q}_{\mathbf{x}}=\frac{\mathrm{d}_{\mathbf{x}}}{\mathrm{E}_{\mathrm{x}}}
$$

The estimate of the probability of surviving from $x$ to $x+1$ is $p_{x}$ where $p_{x}=1-q_{x}$ and the estimate of the (cumulative) probability of surviving from $o$ to $x$ is $P_{x}$ where

$$
P_{x}=p_{0}, p_{1}, p_{2} \ldots p_{x-1} \text { and }\left(P_{o}=1\right)
$$

A graph of $P_{x}$ plotted against $x$ constitutes the survival curve.

A large sample approximate formula for the standard error of $P_{x}$, due to Greenwood (1926), is

$$
\operatorname{SE}\left(P_{x}\right)=P_{x} \sqrt{\sum_{n=0}^{x-1} \frac{d_{n}}{E_{n}\left(E_{n}-d_{n}\right)}}
$$

This may be used for constructing confidence intervals for a particular $\mathbf{P}_{\mathbf{x}}$.

Thus, approximate 95 per cent confidence limits for $P_{x}$ would be

$$
\mathbf{P}_{\mathbf{x}} \pm 1.96 \operatorname{SE}\left(\mathbf{P}_{\mathbf{x}}\right) \text {. }
$$

The difference between two independent survival curves, represented by ${ }^{a} P_{x}$ and ${ }^{b} P_{x}$ (for example, relating to different valves), may be tested by considering the difference between the two cumulative survival probabilities.

An approximate statistical test is to compute $\mathrm{z}$ and compare this with tabulated values of the standard normal distribution, where $\mathrm{z}$ is given by 


$$
\mathrm{z}=\frac{{ }^{a} P_{x}-{ }^{b} P_{x}}{\sqrt{\left(S E\left({ }^{a} P_{x}\right)\right)^{2}+\left(S E\left({ }^{b} P_{x}\right)\right)^{2}}}
$$

Although the time intervals used hitherto have been annual, unequal intervals cause no problems and may be preferable for the early period after operation (Merrell and Shulman, 1955).

Concerning the derivation of $\mathrm{E}_{\mathrm{x}}$ above, we have implicitly assumed that the withdrawals are subject to the same probability of dying subsequent to the last date of contact as those with complete follow-up information. This may be reasonable for those cases who are still in the study population and will be available for future study. There is little reason to believe that this group will differ from those patients who have been observed for longer periods. However, this assumption may be dangerous for the losses and removals, since failure to examine a patient for any reason may be related to the patient's health. This assumption will introduce bias into the estimates $q_{x}$ if the survival experience of the withdrawals is different from those who do not withdraw.

If the withdrawals had been excluded completely from the analysis, this would have involved the more stringent assumption that their survival experience from the date of operation was similar to that for cases with complete follow-up information.

The desirability of minimising the numbers of withdrawals and hence, inter alia, reducing the possible bias of the actuarial estimates is widely recognised.

It is possible to make more sophisticated allowance for the effect of withdrawals on $\mathrm{q}_{\mathrm{x}}$, particularly if the points of withdrawal during an interval are known exactly. But this is unlikely to be the case in surgical applications. In addition, the actuarial approach has the merits of being simple and can be justified intuitively, if, for example, one assumes that the dates of withdrawal are evenly distributed over each interval of follow-up.

In the above derivation, we have also assumed that there is no time-related trend in the probability of dying. The various values of $\mathrm{q}_{\mathrm{x}}$ have been obtained from patients who entered the study at different points in time. We have assumed that these probabilities remain reasonably constant over time; otherwise the qualities computed have no simple interpretation.

The survival rate derived in the previous section is not an estimate of the actual survival rate but of the net or underlying rate (according to the terminology of Grunkemeier et al. (1975)), that would have been obtained if withdrawals were eliminated as a cause of the patient's experience being termi- nated. In the terminology of actuarial mathematics, it is an independent single decrement survival rate (Benjamin and Haycocks, 1970).

\section{INSTANTANEOUS DEATH RATE}

The instantaneous death rate is called the 'force of mortality' in actuarial mathematics and it is a useful index of the intensity of risk. It provides the probability of death occurring in a very small time interval after $x$, given that the patient has survived to time $\mathrm{x}$. We shall denote our estimate of the instantaneous probability of dying at age $x$ by $\mu_{x}$. Assuming that follow-up intervals are of width one year, the value of the force of mortality at age $\mathbf{x}+\frac{1}{2}$ may be estimated by

$$
\mu_{\mathrm{x}+\frac{1}{2}}=\frac{2 \mathrm{q}_{\mathrm{x}}}{2-\mathrm{q}_{\mathrm{x}}}
$$

A large sample approximation to the standard error of $\mu$ has been derived by Gehan (1969), viz

$$
\operatorname{SE}\left(\mu_{\mathrm{x}+\frac{1}{2}}\right)=\frac{\mu_{\mathrm{x}+\frac{1}{2}}}{\sqrt{\mathrm{E}_{\mathrm{x}} \mathrm{q}_{\mathrm{x}}}} \cdot \sqrt{1-\frac{1}{4} \mu^{2} \mathrm{x}+\frac{1}{2}}
$$

A graph of $\mu_{x}$ against $x$ shows whether the risk of death per unit time remains about the same, increases or decreases, or describes a more complex curve. It draws attention to points of time at which the risk changes in intensity by effectively focussing on the gradient of the survival curve.

For intervals of width not equal to one year, the width, $h$, explicitly appears in both the above formulae.

\section{MEDIAN REMAINING LIFETIME}

In nearly all papers discussing life table methods an estimate of the expectations of life or mean lifetime is derived (Irwin, 1947; Kaplan and Meier, 1958; Chiang, 1968). An ambiguity arises in the determination of this estimate if some patients are alive at the end of the study. The expectation of life (which implies an idefinitely long follow-up period) cannot be calculated unless the study period is, at least for some patients, sufficiently long to cover virtually the complete span of life. Otherwise, the mean lifetime is indeterminate and is often estimated as the mean lifetime limited to $\mathrm{N}$ years, where $\mathrm{N}$ is the maximum follow-up period possible within the study. Since this is not easily interpreted, an alternative should be found: we suggest the median remaining lifetime proposed by Gehan (1969).

Estimating the median remaining lifetime is simple, descriptive, and possible if $P_{x}$ is less than 0.5 at some point $\mathrm{x}$, that is the study extends to a point where the probability of survival is less than 0.5 . In elementary statistical textbooks, the median 
is recommended for describing distributions skewed to the right, and so is a natural choice in this situation. The estimate of the median remaining lifetime is $\mathrm{m}_{\mathrm{o}}$ where

$$
\mathrm{m}_{\mathrm{y}}=\mathrm{y}+\frac{\mathrm{P}_{\mathrm{y}}-\frac{1}{2}}{\mathrm{P}_{\mathrm{y}}-\mathrm{P}_{\mathrm{y}}+1}
$$

and $\mathrm{y}$ is the number of complete years up to the point where the survival curve reaches 0.5 , i.e. $\mathbf{P}_{\mathrm{y}}>\frac{1}{2}>\mathbf{P}_{\mathrm{y}+\mathbf{1}}$.

A large sample approximation to the standard error of this estimate is given by

$$
\mathrm{SE}\left(\mathrm{m}_{\mathrm{o}}\right)=\frac{1}{2 \mathrm{P}_{\mathrm{y}} \mathrm{q}_{\mathrm{y}}} \sqrt{\sqrt{\mathrm{E}_{\mathrm{o}}}}
$$

Similarly, the estimate of the median remaining lifetime at time $\mathrm{x}$ after the operation is $\mathrm{m}_{\mathrm{x}}$ where

$$
m_{x}=y-x+\frac{P_{y}-\frac{1}{2} P_{x}}{P_{y}-P_{y+1}}
$$

and $y$ is the number of complete years up to the point where the survival curves reaches $\frac{1}{2} P_{x}$, i.e. $P_{y}>\frac{1}{2} P_{x}>P_{y+1}$.

Similarly,

$$
\mathrm{SE}\left(\mathrm{m}_{\mathrm{x}}\right)=\frac{\mathrm{P}_{\mathrm{x}}}{2 \mathrm{P}_{\mathrm{y}} \mathrm{q}_{\mathrm{y}}} \cdot \frac{1}{\sqrt{\mathrm{E}_{\mathrm{x}}}}
$$

For intervals of width not equal to one year, the width, $h$, explicitly appears in the above formulae.

\section{ALTERNATIVE APPROACHES TO}

ACTUARIAL METHOD

Kaplan and Meier (1958) give a maximum likelihood estimate of the cumulative survival probability called the product limit estimate. This estimate requires knowledge of the exact times of all decrements occurring (for example losses and removals). For large samples, the actuarial method is almost equivalent to the product limit approach.

Several ways in which the partial experience of cases withdrawing can be assigned proper interval credit have been discussed by Littell (1952), Elveback (1958), and Chiang (1968). These methods are more exact than the actuarial method but the latter is conceptually easier, simpler to operate, uses an essentially intuitive approach, and, under certain conditions, the differences may become negligible. For example, Kuzma (1967) compared the actuarial method with that due to Chiang (1968) and found that the differences were negligible for survival rates and their standard errors when the loss rates were under 40 per cent and the proportion of patients withdrawn alive was less than 30 per cent. This emphasises the need for keeping the numbers withdrawing as small as possible.

Among other statistical techniques used in survival analysis, Gehan (1965) proposed an extension of the Wilcoxon non-parametric test for comparing two sets of survival data. This approach has been used by Copeland et al. (1977) for comparing groups of patients having had a valve replacement. In addition, Mantel (1966) has proposed a $\varkappa^{2}$ procedure for comparing two sets of survival data in their entirety. But neither of these methods intrinsically involves the calculation of survival probabilities nor the plotting of survival curves. Both these exercises are natural for the analysis of survival data and the presentation of results. The actuarial method automatically produces these measures in the analysis of survival data.

\section{Appendix II}

The formulae described in this paper will now be used on the information from 140 freeze dried homograft valves over a 13-year follow-up period. Table 1 is the pooled data on these valves: only four of the possible complications have been considered, and some of the valves may have experienced more than one of the complications. Table 1 has been used to construct the life tables $2,3,4,5$, and 6 .

Table 2 is the life table for fatal valve malfunctions. $\mathrm{D}$ represents the deaths, $\mathrm{L}$ the number functioning without complication, and $\mathrm{W}$ includes both the valves with non-fatal complications and

\begin{tabular}{|c|c|c|c|c|c|c|c|c|c|c|c|c|c|}
\hline Years & 1 & 2 & 3 & 4 & 5 & 6 & 7 & 8 & 9 & 10 & 11 & 12 & 13 \\
\hline Valves functioning without complication & 140 & 132 & 122 & 113 & 104 & 95 & 82 & 71 & 58 & 45 & 32 & 14 & 4 \\
\hline Valve related death & 6 & 5 & 5 & 1 & 3 & 6 & 3 & 1 & 3 & 1 & 3 & 0 & 0 \\
\hline Non-fatal complication & 1 & 3 & 4 & 8 & 4 & 3 & 8 & 6 & 5 & 0 & 0 & 1 & 0 \\
\hline Lost/withdrawn & 1 & 2 & 0 & 0 & 2 & 4 & 0 & 6 & 5 & 12 & 15 & 9 & 4 \\
\hline Technical failure & 4 & 7 & 3 & 3 & 0 & 0 & 0 & 0 & 0 & 0 & 0 & 0 & 0 \\
\hline Infection & 2 & 2 & 2 & 1 & 3 & 2 & 1 & 3 & 1 & 0 & 0 & 0 & 0 \\
\hline Degeneration & 0 & 1 & 1 & 5 & 4 & 4 & 8 & 5 & 3 & 1 & 2 & 1 & 0 \\
\hline Thromboembolism & 0 & 0 & 0 & 0 & 0 & 0 & 0 & 0 & 0 & 0 & 0 & 0 & 0 \\
\hline
\end{tabular}
those lost or withdrawn during the year.

Table 1 Pooled data on 140 freeze dried homograft aortic valves used for isolated aortic valve replacement and followed for up to 13 years 
Table 2 Freeze dried homograft valves. Life table for fatal valve malfunctions

\begin{tabular}{|c|c|c|c|c|c|c|c|c|c|c|}
\hline Year & $D$ & $W$ & $L$ & $E$ & $q$ & $p$ & $P$ & $S E P$ & $\mu$ & $S E \mu$ \\
\hline $\begin{array}{l}0 \\
1\end{array}$ & 6 & 2 & 140 & 139 & 0.043 & 0.957 & $\begin{array}{l}1.000 \\
0.957\end{array}$ & 0.017 & & \\
\hline 2 & 5 & 5 & 132 & $129 \cdot 5$ & 0.039 & 0.961 & 0.920 & 0.023 & 0.044 & 0.018 \\
\hline 3 & 5 & 4 & 122 & 120 & 0.041 & 0.959 & 0.881 & 0.027 & 0.040 & 0.018 \\
\hline 4 & 1 & 8 & 113 & 109 & 0.009 & 0.991 & 0.873 & 0.028 & 0.042 & 0.019 \\
\hline 5 & 3 & 6 & 104 & 101 & 0.030 & 0.970 & 0.848 & 0.031 & 0.009 & 0.009 \\
\hline 6 & 6 & 7 & 95 & 91.5 & 0.066 & 0.934 & 0.792 & 0.036 & 0.030 & 0.017 \\
\hline 7 & 3 & 8 & 82 & 78 & 0.036 & 0.964 & 0.761 & 0.038 & 0.063 & 0.026 \\
\hline 8 & 1 & 12 & 71 & 65 & 0.015 & 0.985 & 0.750 & 0.039 & 0.037 & 0.022 \\
\hline 9 & 3 & 10 & 58 & 53 & 0.057 & 0.943 & 0.707 & 0.043 & 0.015 & 0.015 \\
\hline 10 & 1 & 12 & 45 & 39 & 0.026 & 0.974 & 0.689 & 0.044 & 0.059 & 0.034 \\
\hline 11 & 3 & 15 & 32 & $24 \cdot 5$ & $0 \cdot 122$ & 0.878 & 0.605 & 0.052 & 0.026 & 0.026 \\
\hline 12 & 0 & 10 & 14 & 9 & & & 0.605 & 0.052 & $0 \cdot 130$ & 0.075 \\
\hline 13 & 0 & 4 & 4 & 2 & & & 0.605 & 0.052 & 0.00 & \\
\hline
\end{tabular}

$D$, number of deaths; $W$, number withdrawn because of non-fatal complications and those lost to follow-up; $L$, number functioning without complications; $E$, the derived entering numbers; $q$, probability of deaths for a single year; $p$, probability of survival for a single year; $P$, cumulative probability of survival; SE $P$, standard error of $P ; \mu$, instantaneous rate of fatal valve malfunctions; SE $\mu$, standard error of $\mu . \mu$ is calculated for the half year interval and is represented so in Table and Fig. 2.

For year one:

$$
\begin{aligned}
\mathrm{E}_{1} & =\mathrm{L}_{1}-\frac{1}{2}\left(\mathrm{~W}_{1}\right) \\
& =140-\frac{1}{2}(2) \\
& =139 \\
\mathrm{q}_{1} & =\frac{\mathrm{D}_{1}}{\mathrm{E}_{1}} \\
& =\frac{6}{139} \\
& =0.043 \\
\mathrm{p}_{1} & =1-\mathrm{q}_{1} \\
& =1-0.043 \\
& =0.957 \\
\mathrm{P}_{5} & =\mathrm{p}_{0} \times \mathrm{p}_{1} \times \mathrm{p}_{2} \times \mathrm{p}_{3} \times \mathrm{p}_{4} \times \mathrm{p}_{5} \\
& =1.000 \times 0.957 \times 0.961 \times 0.959 \times 0.991 \times 0.970 \\
& =0.848
\end{aligned}
$$

The standard error of $P_{5}$ is calculated from:

$\operatorname{SE}\left(P_{5}\right)=P_{5} \sqrt{\sum_{0}^{4} \frac{d_{n}}{E_{n}\left(E_{n}-d_{n}\right)}}$
The instantaneous death rate, $\mu$, is calculated from:

$$
\begin{aligned}
\mu & =\frac{2 q}{2-q} \\
\mu_{5} & =\frac{2 \times 0.030}{2-0.030} \\
& =\frac{0.060}{1.970} \\
& =0.030
\end{aligned}
$$

The standard error for $\mu_{5}$ is calculated from:

$$
\begin{aligned}
\operatorname{SE}\left(\mu_{5}\right) & =\frac{\frac{\mu_{5}}{\sqrt{\mathrm{E}_{5} \cdot \mathrm{q}_{5}}} \times \sqrt{1-\frac{1}{4}\left(\mu_{5}\right)^{2}}}{} \\
& =\sqrt{\sqrt{101 \cdot 030}} \sqrt{1-\frac{1}{4}(0.030)^{2}} \\
& =\frac{0.030}{\sqrt{3.030}} \times \sqrt{1-\frac{0.009}{4}}
\end{aligned}
$$

$$
\begin{aligned}
& =0.848 \cdot \sqrt{\frac{6}{139(139-6)}+\frac{5}{129 \cdot 5(129 \cdot 5-5)} \times \frac{5}{120(120-5)} \times \frac{1}{109(109-1)} \times \frac{3}{101(101-3)}} \\
& =0.848 \cdot \sqrt{\frac{6}{139 \cdot 133}+\frac{5}{129 \cdot 5 \cdot 124 \cdot 5}+\frac{5}{120 \cdot 115}+\frac{1}{109 \cdot 108}+\frac{3}{101 \cdot 98}} \\
& =0.848 \cdot \sqrt{0.00032+0.00031+0.00036+0.000085+0.00030} \\
& =0.848 \cdot \sqrt{0.00138} \\
& =0.031
\end{aligned}
$$




$$
\begin{aligned}
& =\frac{0.030}{1.741} \times \sqrt{1-0.002} \\
& =0.017 \times 0.999887 \\
& =0.017
\end{aligned}
$$

Table 3 is the life table for the multiple decrement analysis of all valve complications, both fatal and non-fatal. These complications are represented as $D$, and $W$ only represents those valves lost or withdrawn.

For the first year:

$$
\begin{aligned}
\mathrm{E}_{1} & =\mathrm{L}_{1}-\frac{1}{2}(\mathrm{~W}) \\
& =140-\frac{1}{2}(1) \\
& =139 \frac{1}{2}
\end{aligned}
$$

and $q_{1}=\frac{D_{1}}{E_{1}},=\frac{7}{139 \frac{1}{2}}=0.050$

$$
\text { and } \begin{aligned}
\mathrm{p}_{1} & =1-\mathrm{q}_{1}=1-0.050=0.950 \\
\text { and } \mathrm{p}_{5} & =\mathrm{p}_{0} \times \mathrm{p}_{1} \times \mathrm{p}_{2} \times \mathrm{p}_{3} \times \mathrm{p}_{4} \times \mathrm{p}_{5} \\
& =1.00 \times 0.950 \times 0.939 \times 0.926 \times 0.920 \times 0.932 \\
& =0.708
\end{aligned}
$$

The standard error for $P_{5}$ for all malfunctions, the instantaneous malfunction rate $\mu$, and its standard error are calculated as for the fatal malfunctions and all the values are presented in Table 3 .

The values for $P$ from Tables 2 and 3 have been used to construct the actuarial survival curves in Fig. 1, together with the standard errors for $P_{13}$. Similarly, the values for $\mu$ from Tables 2 and 3 have been used for constructing Fig. 2 .

Tables 4, 5, and 6 are the life tables for three types of valve malfunction, respectively, technical error, infection, and degeneration. Each type of malfunc-

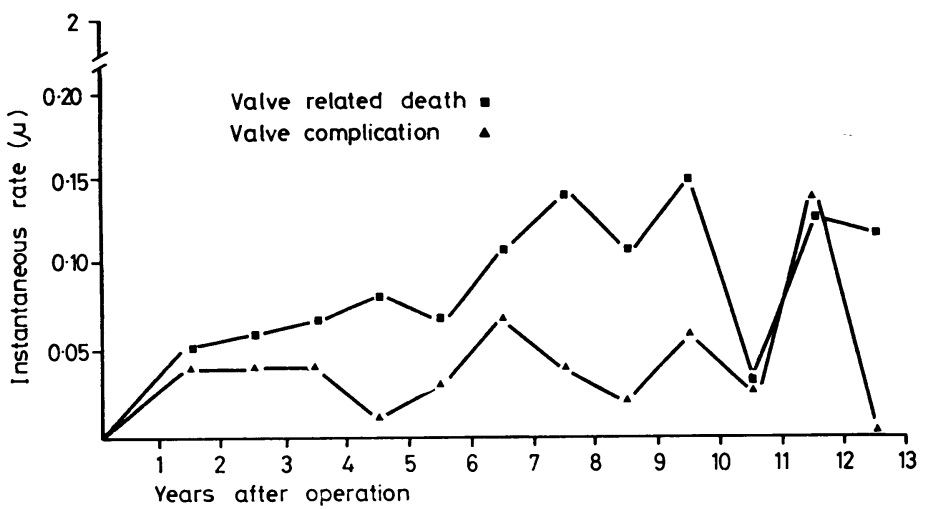

Fig. 2 Freeze dried homograft valves. Instantaneous rate of valve related death or complication.

Table 3 Freeze dried homograft valves. Life table for multiple decrement analysis of all valve complications

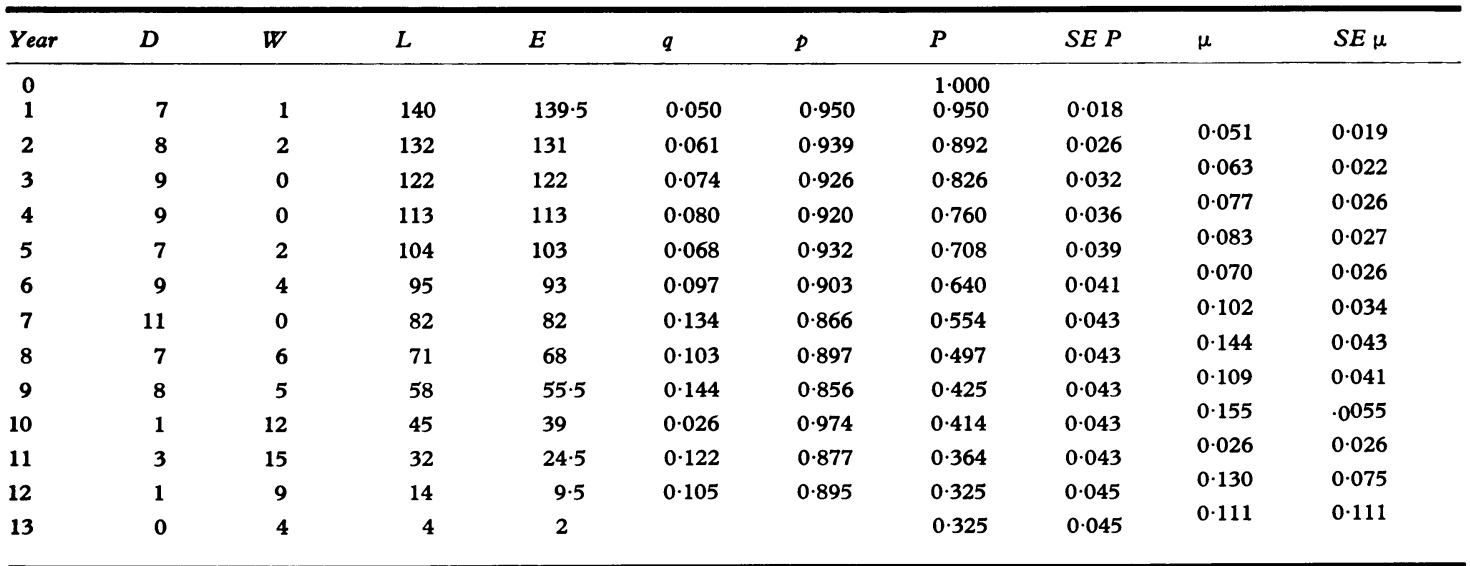

$D$, all the complications; $W$, the valves lost to follow-up; $L$, the number functioning without complication; $E$, the derived entering number; $q$, the probability of any valve complication for a single year; $p$, the probability of freedom from any valve complication for a single year; $P$, the cumulative probability of freedom from any valve complication; $S E P$, the standard error of $P$; $\mu$, the instantaneous rate of valve complication; SE $\mu$, the standard error of $\mu$. $\mu$ calculated to a half year interval and is represented so in Table 3 and Fig. 2 . 
Table 4 Freeze dried homograft valves. Life table for valve malfunctions resulting from technical error only

\begin{tabular}{lllllllllll}
\hline Year & $D$ & $W$ & $L$ & $E$ & $q$ & $p$ & $P$ & $S E P$ & $\mu$ & $S E \mu$ \\
\hline 0 & 4 & 4 & 140 & 138 & 0.029 & 0.971 & 0.971 & 0.014 & 0.029 & 0.014 \\
1 & 7 & 3 & 132 & 130.5 & 0.054 & 0.946 & 0.919 & 0.023 & 0.055 & 0.021 \\
2 & 3 & 6 & 122 & 119 & 0.025 & 0.975 & 0.896 & 0.026 & 0.025 & 0.014 \\
3 & 3 & 6 & 113 & 110 & 0.027 & 0.973 & 0.871 & 0.029 & 0.027 & 0.015 \\
5 & 0 & & & & & & & & &
\end{tabular}

$D$, the number of valves with a technical error; $W$, the number of valves withdrawn because of other complications or lost to follow-up; $L$, the number functioning without technical errors; $E$, the derived entering number; $q$, the probability of a technical error for a single year; $p$, the probability of freedom from a technical error for a single year; $P$, the cumulative probability of freedom from a technical error; SE P, the standard error of $P ; \mu$, the instantaneous rate of technical error; SE $\mu$, the standard error of $\mu$; $\mu$, calculated to the half year and is represented so in Table 4 and Fig. 4.

Fig. 3 Individual complication ree function curves of freeze dried homograft valves. Probabilities of freedom from thromboembolism, technical problems, infection, or degeneration.

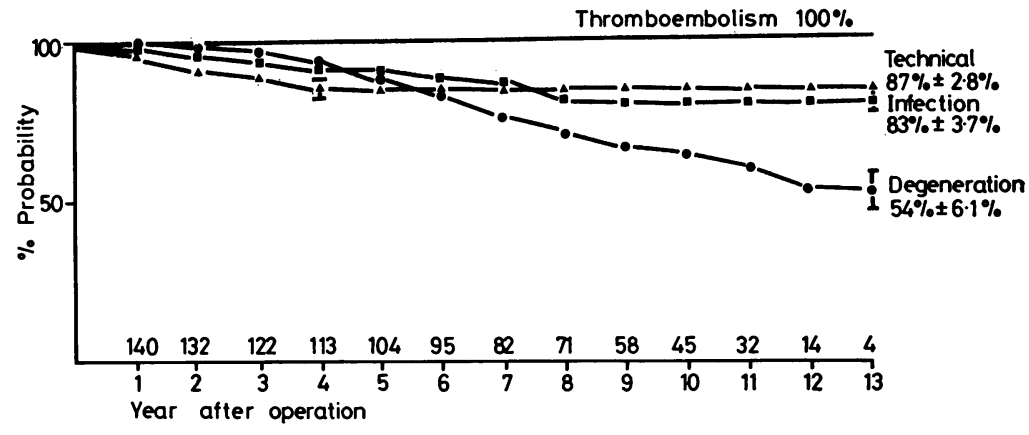

Table 5 Freeze dried homograft valves. Life table for valve malfunction resulting from infection only

\begin{tabular}{llllllllllll}
\hline Year & $D$ & $W$ & $L$ & $E$ & $q$ & $p$ & $P$ & $S E P$ & $\mu$ & $S E \mu$ \\
\hline 0 & 2 & 6 & 140 & 137 & 0.014 & 0.985 & 0.985 & 0.010 & 0.014 & 0.010 \\
1 & 2 & 8 & 132 & 128 & 0.016 & 0.984 & 0.970 & 0.014 & 0.016 & 0.011 & 0.012 \\
2 & 2 & 7 & 122 & 118.5 & 0.017 & 0.983 & 0.954 & 0.018 & 0.017 & 0.012 \\
3 & 1 & 8 & 113 & 109.5 & 0.009 & 0.991 & 0.945 & 0.020 & 0.009 & 0.009 \\
4 & 3 & 6 & 104 & 101 & 0.030 & 0.970 & 0.917 & 0.025 & 0.030 & 0.017 \\
5 & 2 & 11 & 95 & 89.5 & 0.022 & 0.978 & 0.896 & 0.028 & 0.022 & 0.016 \\
6 & 1 & 10 & 82 & 77 & 0.013 & 0.987 & 0.885 & 0.029 & 0.013 & 0.013 \\
7 & 3 & 10 & 71 & 66 & 0.045 & 0.954 & 0.844 & 0.035 & 0.046 & 0.027 \\
8 & 1 & 12 & 58 & 52 & 0.019 & 0.981 & 0.828 & 0.037 & 0.019 & 0.019 \\
9 & 0 & & & & & & & & & &
\end{tabular}

$D$, the number of infected valves; $W$, the number withdrawn because of other malfunctions or lost to follow-up; $L$, the number of valves functioning without infection; $E$, the derived entering number; $q$, the probability of infection for a single year; $p$, the probability of freedom from infection; $P$, the cumulative probability of freedom from infection; SE $P$, the standard error of $P$; $\mu$, the instantaneous rate of infection; SE $\mu$, the standard error of $\mu ; \mu$ is calculated to the half year and is represented so in Table 5 and Fig. 4 . 
Table 6 Freeze dried homograft valves. Life table for valve malfunction caused by degeneration only

\begin{tabular}{|c|c|c|c|c|c|c|c|c|c|c|}
\hline Year & $D$ & $W$ & $L$ & $E$ & $q$ & $p$ & $P$ & $S E P$ & $\mu$ & $S E \mu$ \\
\hline 0 & & & & & & & 1.000 & & & \\
\hline 1 & 0 & 8 & 140 & 136 & 0 & 1.0 & $1 \cdot 0$ & & 0.000 & 0 \\
\hline 2 & 1 & 9 & 132 & $127 \cdot 5$ & 0.008 & 0.992 & 0.992 & 0.007 & & \\
\hline 3 & 1 & 8 & 122 & 118 & 0.008 & 0.992 & 0.984 & 0.011 & 0.008 & 0.008 \\
\hline 4 & 5 & 4 & 113 & 111 & 0.045 & 0.955 & 0.939 & 0.022 & 0.008 & 0.008 \\
\hline 5 & 4 & 5 & 104 & 101.5 & 0.039 & 0.961 & 0.902 & 0.027 & 0.046 & 0.021 \\
\hline 6 & 4 & 9 & 95 & 90.5 & 0.044 & 0.956 & 0.863 & 0.032 & 0.040 & 0.020 \\
\hline 7 & 8 & 3 & 82 & 80.5 & 0.099 & 0.901 & 0.777 & 0.040 & 0.045 & 0.023 \\
\hline 8 & 5 & 8 & 71 & 67 & 0.075 & 0.925 & 0.719 & 0.044 & 0.104 & 0.037 \\
\hline 9 & 3 & 10 & 58 & 53 & 0.057 & 0.943 & 0.678 & 0.047 & 0.078 & 0.035 \\
\hline 10 & 1 & 12 & 45 & 39 & 0.026 & 0.974 & 0.661 & 0.048 & & 0.034 \\
\hline 11 & 2 & 16 & 32 & 24 & 0.083 & 0.917 & 0.606 & 0.052 & 0.087 & 0020 \\
\hline 12 & 1 & 9 & 14 & 9.5 & $0 \cdot 105$ & 0.895 & 0.542 & 0.061 & 0.111 & $0 \cdot 111$ \\
\hline 13 & 0 & 4 & 4 & 2 & 0 & 1.00 & 0.542 & 0.061 & 0.000 & 0 \\
\hline
\end{tabular}

$D$, the number of valve malfunctions with degeneration; $W$, the number of valves withdrawn because of other malfunctions or lost to follow-up; $L$, the number of valves functioning without degeneration; $E$, the derived entering number; $q$, the probability of degeneration for a single year; $p$, the probability of freedom from degeneration for a single year; $P$, the cumulative probability of freedom from degeneration; SE $P$, the standard error of $P ; \mu$, the instantaneous rate of degeneration; SE $\mu$, the standard error of $\mu ; \mu$ is calculated to the half year and is represented so in Table 6 and Fig. 4.

tion is entered as $\mathrm{D}$, and the value for $\mathrm{W}$ is calculated from:

$$
\mathrm{W}_{1}=\mathrm{L}_{1}-\left(\mathrm{L}_{2}+\mathrm{D}_{1}\right)
$$

that is for year one from Table 4

$$
\begin{aligned}
\mathrm{W}_{1} & =140-(132+4) \\
& =140-136 \\
& =4 \\
\mathrm{E}_{1} & =\mathrm{L}_{1}-\frac{1}{2}\left(\mathrm{~W}_{1}\right) \\
& =140-\frac{1}{2}(4) \\
& =138 \\
\mathrm{q}_{1} & =\frac{\mathrm{D}_{1}}{\mathrm{E}_{1}} \\
& =\frac{4}{138} \\
& =0.029 \\
\mathrm{p}_{1} & =1-\mathrm{q}_{1} \\
& =0.971
\end{aligned}
$$

and $P$ and $\mu$ are calculated as before, with their respective standard errors, to give all the entries in Tables 4, 5, and 6 .

The values of $P$ from Tables 4, 5, and 6 and for thromboembolism have been used for constructing the individual event-free curves in Fig. 3 and the values of $\mu$ have been used for Fig. 4 .

The median remaining lifetime can be calculated from the data in Table 2 using the formulae.
The median remaining lifetime free of complication at time $0, \mathrm{~m}_{0}$, is calculated from:

$$
\begin{aligned}
\mathrm{m}_{0} & =\mathrm{y}+\frac{\mathrm{P}_{\mathrm{y}-\frac{1}{2}}}{\mathrm{P}_{\mathrm{y}}-\mathrm{P}_{\mathrm{y}+1}} \\
\mathrm{~m}_{0} & =7+\frac{0.554-0.500}{0.554-0.497} \\
& =7.947 \text { years }
\end{aligned}
$$

and the standard error is calculated from

$$
\begin{aligned}
\operatorname{SE}\left(\mathrm{m}_{0}\right) & =\frac{1}{2 \cdot \mathrm{P}_{\mathrm{y}} \cdot \mathrm{q}_{\mathrm{y}}} \times \frac{1}{\sqrt{\overline{\mathrm{E}_{\mathrm{o}}}}} \\
& =\frac{1}{2 \times 0.554 \times 0.134} \times \frac{1}{\sqrt{139.5}} \\
& =6.735 \times 0.085 \\
& =0.570
\end{aligned}
$$

The median remaining life time free of complication at year five, $\mathrm{m}_{5}$, is calculated from

$$
\begin{aligned}
\mathrm{m}_{\mathrm{x}} & =\mathrm{y}-\mathrm{x}+\frac{P_{\mathrm{y}}-\frac{1}{2} \mathrm{P}_{\mathrm{x}}}{\mathrm{P}_{\mathrm{y}}-\mathrm{P}_{\mathrm{y}+1}} \\
\mathrm{~m}_{5} & =11-5+\frac{0.364-0.355}{0.364-0.325} \\
& =6.231 \text { years }
\end{aligned}
$$




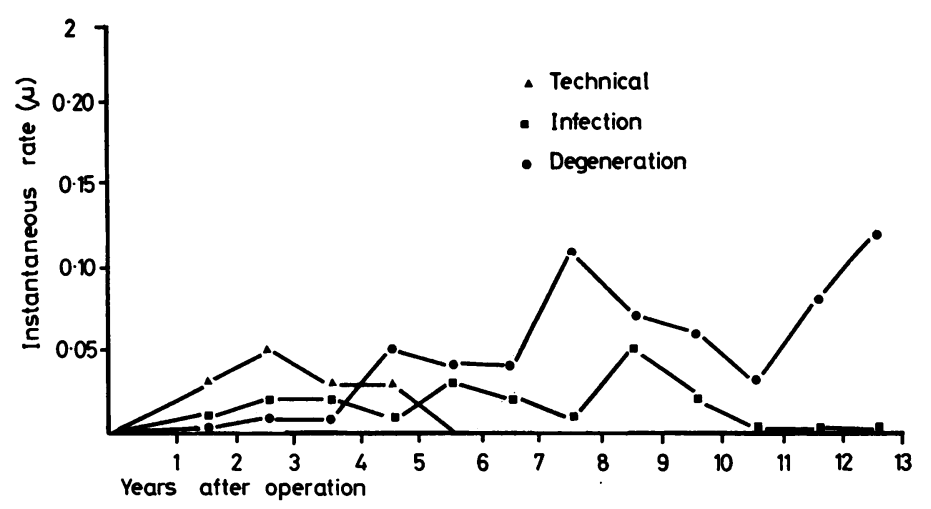

Fig. 4 Freeze dried homograft valves. Instantaneous rate of technical problems, infection, or degeneration. and the standard error is calculated from:

$$
\begin{aligned}
\operatorname{SE}\left(m_{x}\right) & =\frac{P_{x}}{2 \cdot P_{y} \cdot q_{y}} \times \frac{1}{\sqrt{\mathrm{E}_{x}}} \\
\operatorname{SE}\left(m_{5}\right) & =\frac{0.708}{2 \times 0.364 \times 0 \cdot 122} \times \frac{1}{\sqrt{104}} \\
& =7.97 \times 0.098 \\
& =0.782
\end{aligned}
$$

This Appendix shows how follow-up information can be used to provide accurate data on an actuarial basis for the evaluation and comparison of different cardiac valve replacements.

\section{References}

Anderson, R., Bonchek, L. I., Grunkemeier, G., Lambert, L., and Starr, A. (1974). The analysis and presentation of surgical results by actuarial methods. Fournal of Surgical Research, 16, 224-230.

Axtell, L. (1963). Computing survival rates for chronic disease patients: a simple procedure. Fournal of the American Medical Association, 186, 1125-1128.

Benjamin, B., and Haycocks, H. (1970). The Analysis of Mortality and Other Actuarial Statistics. Cambridge University Press, London.

Berkson, J., and Gage, R. (1950). Calculation of survival rates for cancer. Proceedings of the Staff Meetings of the Mayo Clinic, 25, 270-286.

Chiang, C. (1968). Computing risks. In Introduction to Stochastic Processes in Biostatistics, pp. 242-263, ed C. Chiang. J. Wiley, New York.

Copeland, J., Griepp, R. B., Stinson, E. B., and Shumway, N. E. (1977). Long-term follow up after isolated aortic valve replacement. Fournal of Thoracic and Cardiovascular Surgery, 74, 875-889.

Cutler, S., and Ederer, F. (1958). Maximum utilization of the life table method in analyzing survival. Fournal of Chronic Diseases, 8, 699-712.
Elveback, L. (1958). Estimation of survivorship in chronic disease: the 'actuarial' method. Fournal of the American Statistical Association, 53, 420-440.

Frost, W. (1933). Risk of persons in familial contact with pulmonary tuberculosis. American fournal of Public Health, 23, 426-432.

Gehan, E. A. (1965). A generalized Wilcoxon test for comparing arbitarily singly-censored samples. Biometrika, 52, 203-223.

Gehan, E. (1969). Estimating survival functions from the life table. Fournal of Chronic Diseases, 21, 629-644.

Greenwood, M. (1926). The 'errors of sampling' of the survivorship tables. A Report on the Natural Duration of Cancer. Reports on Public Health and Medical Subjects, No. 33, Appendix I, pp. 23-25. H.M.S.O., London.

Grunkemeier, G., Lambert, L., Bonchek, L. I., and Starr, A. (1975). An improved statistical method for assessing the results of operation. Annals of Thoracic Surgery, 20, 289298.

Irwin, J. O. (1947). The standard error of estimate of an expectation of life, with special reference to expectations of tumourless life in experiments with mice. Fournal of Hygiene, 49, 188-189.

Kaplan, E., and Meier, P. (1958). Nonparametric estimation from incomplete observations. Fournal of the American Statistical Association, 53, 457-481.

Karn, N. (1931). An inquiry into various death rates and the comparative influence of certain diseases on the duration of life. Annals of Eugenics, 4, 279-326.

Kuzma, J. (1967). A comparison of two life table methods. Biometrics, 23, 51-64.

Littell, A. (1952). Estimation of the T-year survival rate from follow up studies over a long period of time. Human Biology, 24, 87-116.

Mantel, N. (1966). Evaluation of survival data and two new rank order statistics arising in its consideration. Cancer Chemotherapy Reports, 50, 163-170.

Merrell, M., and Shulman, L. (1955). Determination of prognosis in chronic disease, illustrated by systemic lupus erythematosus. Fournal of Chronic Diseases, 1, 12-32.

Requests for reprints to Dr Endre Bodnar, Department of Surgery, Cardiothoracic Institute, 2 Beaumont Street, London W1N 2DX. 